\title{
Comparative evaluation of the osteogenic capacity of human mesenchymal stem cells from bone marrow and umbilical cord tissue
}

\author{
QIAN WANG, GANG ZHAO, ZIJUN XING, JUMING ZHAN and JIE MA \\ Institute of Biomedical Science, Tianjin Kang Ting Biological Engineering Co., Ltd., Tianjin 300385, P.R. China
}

Received April 23, 2018; Accepted September 27, 2018

DOI: $10.3892 /$ etm.2018.6975

\begin{abstract}
Mesenchymal stem cells (MSCs) have been extensively investigated in the field of regenerative medicine. Human bone MSCs (BMSCs) have become a common type of seed cell for bone tissue engineering. However, the viability and cell number of BMSCs are negatively correlated with donor age, and as the extraction process is painful, this method has not been widely used. As human umbilical cord MSCs (UCMSCs) may be harvested inexpensively and inexhaustibly, the present study evaluated and compared the regenerative potential of UCMSCs and BMSCs to determine whether UCMSCs may be used as a novel cell type for bone regeneration. In the present study, the proliferation and osteogenic capacity of BMSCs and UCMSCs was compared in vitro. BMSCs and UCMSCs were respectively combined with biofunctionalized macroporous calcium phosphate cement, and their bone regenerative potentials were determined by investigating their capacity for ectopic bone formation in a nude mouse model as well as their efficacy in a rat model of tibia bone defect. The extent of bone regeneration was examined by $\mathrm{X}$-ray, histological and immunohistochemical analyses. The results revealed that UCMSCs exhibited a good osteogenic differentiation potential, similarly to that of BMSCs, and that UCMSCs were able to contribute to the regeneration of bone and blood vessels. Furthermore, no significant differences were identified between BMSCs and UCMSCs in terms of their bone regenerative effect.
\end{abstract}

\section{Introduction}

Bone defects, which may be caused by infections, trauma, tumors or various congenital diseases, have become a major challenge in the field of orthopedics (1). Current methods

Correspondence to: Dr Jie Ma, Institute of Biomedical Science, Tianjin Kang Ting Biological Engineering Co., Ltd., 126 Youyinan Road, Tianjin 300385, P.R. China

E-mail: biocare@ktibs.com

Key words: bone marrow mesenchymal stem cells, umbilical cord mesenchymal stem cells, bone defect, calcium phosphate cement, osteogenic differentiation for treating bone defects include autografts, allograft bone transplantation, tissue engineering techniques and gene therapy. Autografts are recognized as the ideal bone graft material, although these are associated with a number of drawbacks, including a limited supply, pain at the donor site and complications; furthermore, the sources of allogeneic bone are limited, and a risk of infectious diseases and varying degrees of immune response are present (2). Bone tissue engineering, which comprises signaling molecule scaffold materials and seed cells, holds promise for bone defect repair. Mesenchymal stem cells (MSCs) are adult stem cells that are regarded as ideal seed cells due to their low immunogenicity, as well as a high self-replicative ability and multi-directional differentiation potential $(3,4)$. These stem cells may develop into multiple mesodermal lineages, including bone and cartilage (5). Over the course of the past few decades, MSCs have been isolated from various types of tissue, including the bone marrow, umbilical cord blood, umbilical cord tissue, placental tissue and adipose tissue (6). However, BMSCs and UCMSCs remain the major sources of MSCs, particularly in autologous cell-based therapies, due to the ease of harvest and potential autologous application (6-8). Indeed, BMSCs are recognized as the ideal seed cells for bone tissue engineering therapies, and have been successfully applied in the clinic. However, the negative correlation between the viability and number of BMSCs with donor age limits their applicability. Previous studies have indicated that UCMSCs may be easily isolated, have a low immunogenicity and are able to differentiate into mesenchymal lineages; therefore, UCMSCs have been considered to have a high potential to be used as seed cells $(9,10)$. To confirm whether UCMSCs may be employed as an alternative type of seed cell for bone regeneration, the present study compared BMSCs and UCMSCs in vitro and in ectopic bone formation and tibia bone defect models.

In addition to the seed cells, the signaling molecule scaffold materials also serve an essential role in cell-based bone regeneration. These scaffolds are named 'biomimetic', as they provide a suitable microenvironment for cell attachment, growth, differentiation and reproduction $(11,12)$. In vivo studies have demonstrated de novo bone formation or mineral deposition in MSC-implanted scaffolds, as well as the direct involvement of transplanted cells in bone regeneration $(6,12,13)$. Numerous studies have focused on the use 
of different types of scaffold as basic tools for regenerative medicine $(10,14)$. Biofunctionalized macroporous calcium phosphate cement (CPC) has demonstrated properties of excellent biocompatibility, osteoconductivity, in situ hardening and molding capabilities, as well as injectability, and may be resorbed and replaced by newly formed bone in vivo $(12,15,16)$. $\mathrm{CPC}$ has the potential to fuse directly with the host bone and to guide osteogenesis. However, to date, a comparison of UCMSCs and BMSCs seeded on CPC scaffolds for bone regeneration has not been provided, to the best of our knowledge.

In the present study, the osteogenic capacities of BMSCs and UCMSCs in vitro were investigated by seeding them on $\mathrm{CPC}$, and a comparison of their osteogenic potential was made using ectopic bone formation and tibia defect models in vivo.

\section{Materials and methods}

Animals. A total of 27 male Sprague Dawley (SD) rats (220-240 g; 6-8 weeks) and 27 male Balb/c nude mice (18-22 g; 6-8 weeks) were purchased from Beijing Vital River Laboratory Animal Technology Co., Ltd. (Beijing, China). The SD rats received free access to food and water and were housed at a temperature of $20-26^{\circ} \mathrm{C}$, a humidity of $50-60 \%$ and a $12 \mathrm{~h}$ light/dark cycle. The Balb/c nude mice were put in individual ventilated cages for mating in the barrier system with ad libitum access to standard murine food and water. The temperature, humidity and light/dark cycle were the same as the rats as mentioned above.

The Institute of Radiation Medicine of the Chinese Academy of Medical Sciences (Tianjin, China) provided an animal experiment platform in which the animal experiments were performed. The protocol was approved by the medical ethics committee of the Institute of Radiation Medicine Chinese Academy of Medical Sciences (Tianjin, China).

Culture of BMSCs and UCMSCs. BMSCs and UCMSCs were purchased from Cyagen Biosciences, Inc. (Guangzhou, China). Cells were revived and seeded in culture flasks, and maintained at $37^{\circ} \mathrm{C}$ in humidified atmosphere containing $5 \% \mathrm{CO}_{2}$ in Dulbecco's modified Eagle's medium (DMEM)/ F-12 (HyClone (HM $^{\mathrm{TM}}$ GE Healthcare, Logan, UT, USA) containing $10 \%$ fetal bovine serum (FBS; Lanzhou Bailing Biotechnology Co., Ltd., Lanzhou, China) and $1 \%$ penicillin and streptomycin (P/S; North China Pharmaceutical Group Co., Ltd., Hebei, China). Cells were allowed to expand until they reached $80 \%$ confluence. Passage- 3 cells were used in the present study.

Routine characterization of the BMSCs and UCMSCs was performed according to the manufacturer's protocol, as described previously $(17,18)$. After reaching $80 \%$ confluence, the cells were treated with $0.25 \%$ trypsin-EDTA. Cells were incubated with fluorochrome-conjugated primary antibodies against CD34 (cat. no. 555822), CD44 (cat. no. 555479), CD45 (cat. no. 555483), CD73 (cat. no. 550257), CD90 (cat. no. 555596) and CD105 (cat. no. 560539; all from BD Biosciences, San Jose, CA, USA) at $4^{\circ} \mathrm{C}$ for $30 \mathrm{~min}$. The samples were subsequently measured on a flow cytometer (BD FACSCanto II; BD Biosciences) and data was analyzed with FlowJo Version 7.6 software (Tree Star, Inc., Ashland, OR, USA).
A Cell Counting Kit-8 (CCK-8; Tongren Shanghai Co., Shanghai, China) was used to evaluate the proliferation of BMSCs and UCMSCs. In brief, $2 \times 10^{3}$ cells were seeded onto 96-well plates and cultured for 8 days. Every day, designated wells were stained with $\mathrm{CCK}-8$ reagent according to the manufacturer's protocols, the absorbance at $450 \mathrm{~nm}$ was determined using a microplate reader.

Osteogenic differentiation. BMSCs and UCMSCs at passage 3 were cultured in 6-well plates with DMEM/F-12 medium. The next day, the media in the experimental groups were replaced with osteo-inductive medium (OIM) consisting of $50 \mu \mathrm{M}$ ascorbate-2-phosphate, $10 \mathrm{mM} \beta$-glycero-phosphate and 10 nM dexamethasone (all from Sigma-Aldrich; Merck KGaA, Darmstadt, Germany) and control groups were replaced with DMEM/high glucose medium (HyClone ${ }^{\mathrm{TM}}$; GE Healthcare) with 10\% FBS (6). Alkaline phosphatase (ALP) staining was performed using the Alkaline Phosphatase kit (cat. no. 86R-1KT; Sigma-Aldrich; Merck KGaA) according to the manufacturer's instructions at day 9. Staining with Alizarin red S (cat. no. A5533, Sigma-Aldrich; Merck KGaA) was performed at day 18. Cells were washed with PBS, fixed with 95\% ethanol at room temperature for $15 \mathrm{~min}$ and stained with $0.1 \%$ Alizarin Red S (pH 4.2; Sigma-Aldrich; Merck KGaA) at $37{ }^{\circ} \mathrm{C}$ for $30 \mathrm{~min}$. The medium was replaced every 3 days during culture.

Engineering of cell-scaffold constructs. BMSCs and UCMSCs at passage 3 were used in the subsequent experiments. First, the CPC scaffolds (Shanghai Rebone Biomaterials Co., Ltd., Shanghai, China) were cut into an $8 \mathrm{~mm}$ diameter round sheet with an average thickness of $2 \mathrm{~mm}$, or to the size of $2 \times 2 \times 6 \mathrm{~mm}$. The CPCs were placed at the bottom of 24-well plates in a $100-\mu 1$ suspension $\left(1 \times 10^{6}\right.$ cells $\left./ \mathrm{ml}\right)$ of BMSCs or UCMSCs. Subsequently, the scaffold and cell complexes were cultivated at $37^{\circ} \mathrm{C}$ with DMEM/F-12 for $24 \mathrm{~h}$. As a control, certain CPC pieces were immersed in medium lacking cells prior to transplantation into the animal model.

Tibia defect model. A total of 27 mature SD rats were used for the generation of the tibia bone defect model as described previously $(19,20)$. In brief, general anesthesia was performed by administering sodium pentobarbital $(40 \mathrm{mg} / \mathrm{kg}$ body weight; intraperitoneal injection; Merck KGaA), and a $1-2 \mathrm{~cm}$ skin incision was made to separate and expose the tibia following disinfection. A unilateral $2 \times 2 \times 6-\mathrm{mm}$ defect was created in the middle part of the tibia. Rats were then randomly divided into three groups ( $n=3$ per group): The BMSCs+CPC scaffold, the UCMSCs+CPC scaffold and the control group. For the control group, the right side of the rats were implanted with the cell-free CPC scaffold, whereas the left side remained untreated. Finally, the surgical site was sutured. The grafts from each group were harvested at 4, 6 and 8 weeks after the surgery, and these were then evaluated using X-ray analysis, as well as histological and immunohistochemical techniques.

Ectopic bone formation. A total of $27 \mathrm{BALB} / \mathrm{c}$-nude mice aged 6-8 weeks were used for the ectopic bone formation experiment (21). Mice were randomly divided into three groups $(n=3$ 
per group): BMSCs+CPC scaffold, UCMSCs+CPC scaffold and CPC scaffold (cell-free). Following anesthesia, an incision was made in the dorsal side of the nude mouse and the scaffold construct ( $8 \mathrm{~mm}$ in diameter) was implanted subcutaneously. The wound was then closed in layers. The grafts were harvested at 4,6 and 8 weeks following surgery and fixed in $4 \%$ paraformaldehyde.

Histological observations. Grafts were embedded in paraffin and cut into 5- $\mu \mathrm{m}$ sections. These specimens were divided into two parts. One part was stained with H\&E, as described previously (22), whereas the other part was used for immunofluorescence experiments. Osteopontin (OPN; cat. no. sc-21742; 1:50; Santa Cruz Biotechnology, Inc., Dallas, TX, USA), type I collagen (COL-1; cat. no. ab34710; 1:200; Abcam, Cambridge, UK), Runt-related transcription factor 2 (Runx2; cat. no. sc-10758; 1:50; Santa Cruz Biotechnology, Inc.) and vascular endothelial growth factor (VEGF; cat. no. ab46154; 1:200; Abcam) were assessed according to protocols of previous studies $(20,23)$. Immunohistochemistry was performed using the streptavidin-peroxidase method (SPlink Detection kit; cat. no. SP-9001; OriGene Technologies, Inc., Rockville, MD, USA) according to the manufacturer's protocol. The kit comprised Reagent A (goat serum), B (goat anti-rabbit immunoglobulin G) and $\mathrm{C}$ (horseradish peroxidase). Endogenous peroxidase activity was quenched using $1 \% \mathrm{H}_{2} \mathrm{O}_{2}$ /methanol for $10 \mathrm{~min}$ and antigen retrieval was performed with citrate buffer in the microwave for $10 \mathrm{~min}$. Slides were blocked with reagent A at room temperature for $20 \mathrm{~min}$ and stained with the aforementioned primary antibodies overnight at $4^{\circ} \mathrm{C}$. Reagent $\mathrm{B}$ was then added and incubated at $37^{\circ} \mathrm{C}$ for $20 \mathrm{~min}$. Reagent $\mathrm{C}$ was added and incubated for $15 \mathrm{~min}$ at $37^{\circ} \mathrm{C}$. DAB (cat. no. ZLI-9019; OriGene Technologies, Inc.) was used for further staining at room temperature for $6 \mathrm{~min}$. Finally, sections were counterstained with hematoxylin for $1 \mathrm{~min}$ at room temperature and observed under an optical microscope (magnification, x100; Nikon Ti-S; Nikon Corporation, Tokyo, Japan) (20). In order to semi-quantitatively analyze the immunoreactivity of OPN, COL-1 and VEGF, the H-score was determined. The H-score was calculated by combining the percentage of immunoreactive cells (\%) with an estimate of the staining intensity $(0,1,2$ or 3$)$, as described previously (24). An H-score of 9-12 was considered to represent strong immunoreactivity ('+++'), 5-8 was considered moderate ('++'), 1-4 was considered weak ('+') and 0 was scored as negative ('-') $(25,26)$.

Statistical analysis. Data were analyzed using SPSS 19.0 software (IBM Corp., Armonk, NY, USA). Values are expressed as the mean \pm standard deviation. One-way analysis of variance and Tukey's post hoc test were used to determine significant differences between the groups. $\mathrm{P}<0.05$ was considered to indicate a statistically significant difference.

\section{Results}

Expansion and characterization of the cells. Assessment of the growth activities of BMSCs and UCMSCs indicated that they exhibited a similar proliferation rate within all phases of the growth period. In each of the two cases, cells proliferated slowly at first, but then entered the exponential growth

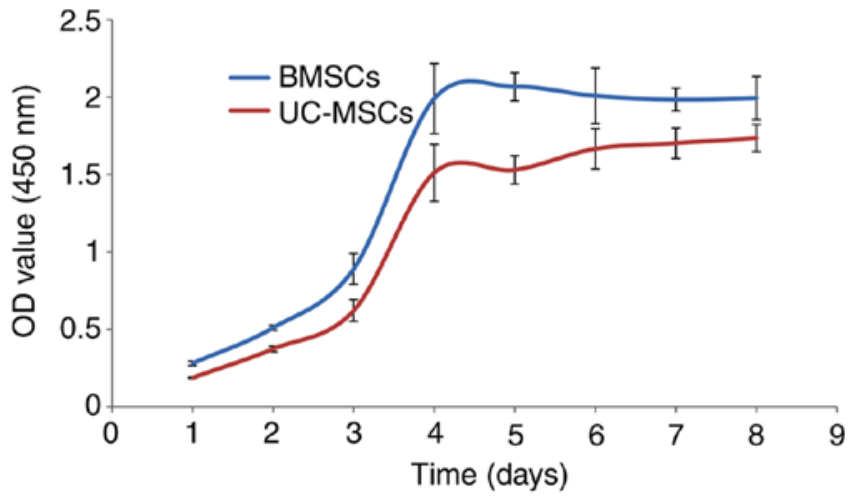

Figure 1. Growth curves of BMSCs and UCMSCs. OD, optical density; BMSCs, bone marrow mesenchymal stem cells; UCMSCs, umbilical cord mesenchymal stem cells.

phase, which continued for 3-5 days. Finally, the cells became confluent and reached the period of stagnation (Fig. 1).

Flow cytometry was subsequently performed to identify the typical cell-surface markers for MSCs. The results indicated that BMSCs and UCMSCs were positive for the MSC markers CD44, CD90 and CD105, but negative for the markers CD14, CD34 and CD45 (Fig. 2). These results suggested that the expression of cell markers was similar for BMSCs and UCMSCs, and that the expression patterns conformed with the typical surface markers for MSCs.

Osteogenesis of BMSCs and UCMSCs in vitro. After having been cultured in OIM for 9 days, the expression levels of ALP were high in the BMSCs and the UCMSCs. After 18 days of culture in OIM, mineralized nodules were observed in the BMSCs and UCMSCs, as demonstrated by Alizarin Red staining (Fig. 3).

Bone regeneration using the tibia defect model. To compare the effects of BMSCs and UCMSCs on bone repair, the SD rat tibia defect model was constructed. The bone structure in the defective area was observed by X-ray analysis at 4,6 and 8 weeks after surgery. Fewer, or no new osseous were observed in the defect area in control group (Fig. 4).

In addition, in the area of the defect, flaky cartilage, bone-like matrixes and lacunae were observed in numerous instances. A certain amount of fibrous tissue and vascular hyperplasia were also observed. Lamellar bone was identified on each side of the bone, which was densely arranged and close to having reached maturity. The surface of the lamellar bone was lined with osteoblasts, and a normal bone marrow cavity was observed at 8 weeks following the surgery in the BMSCs+CPC and UCMSCs+CPC groups. However, in the CPC group, only a small amount of vascular hyperplasia was observed, a large number of neutrophlis had infiltrated into the area of the defect, and comparatively less mature cancellous bone and normal bone tissue was observed in the surrounding area on H\&E staining (Fig. 5A).

At 4 weeks after the surgery, the expression of OPN and COL-1 was detected in the BMSCs+CPC and UCMSCs+CPC groups, although the staining lacked structure and definition (Fig. 5B and C). At 8 weeks after the surgery, a markedly 

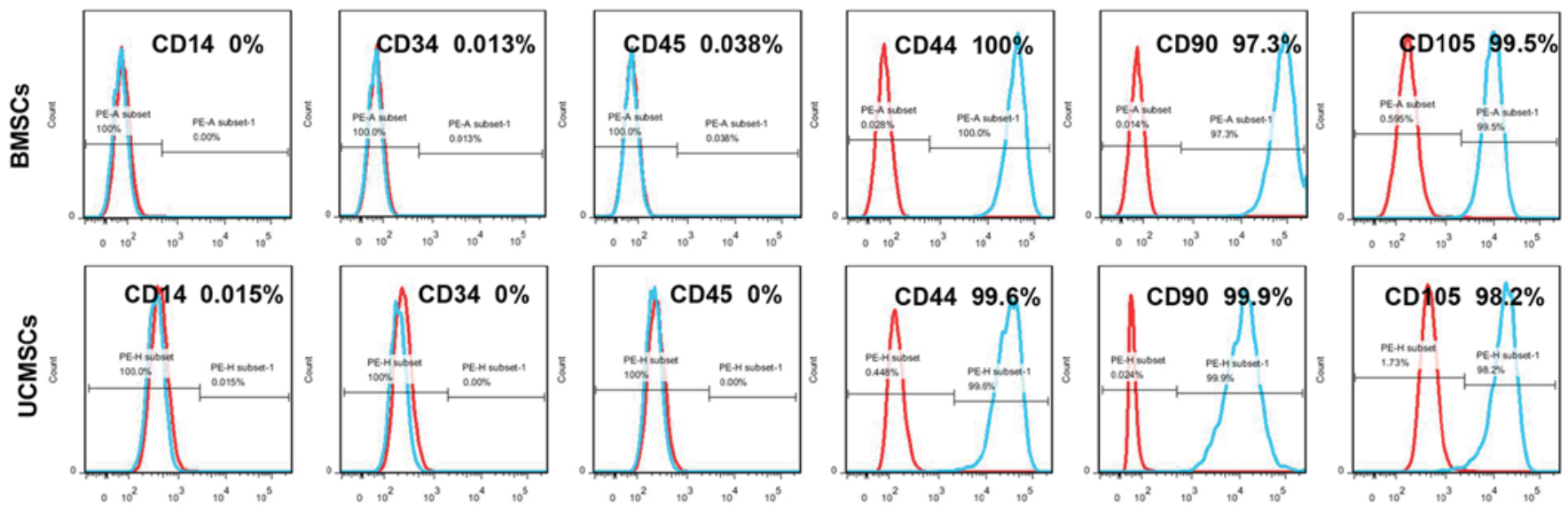

Figure 2. Characterization of cell surface markers of BMSCs and UCMSCs. The red line represents the isotype control and the blue line represents the sample. BMSCs, bone marrow mesenchymal stem cells; UCMSCs, umbilical cord mesenchymal stem cells; PE, phycoerythrin.
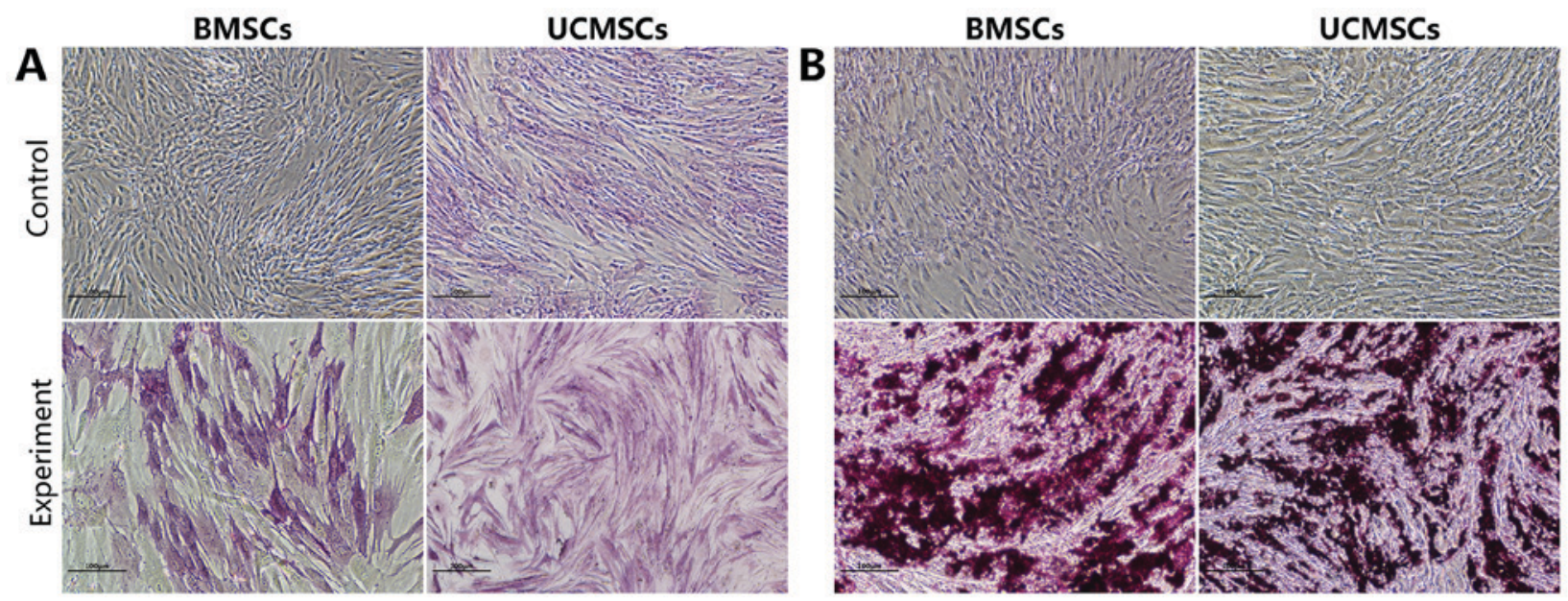

Figure 3. Comparison of the osteogenic abilities of BMSCs and UCMSCs was performed using (A) alkaline phosphatase staining and (B) alizarin red S staining at 9 and 18 days respectively. The experiment group was cultured in osteo-inductive medium and the control was cultured in Dulbecco's modified Eagle's medium/F-12. Scale bar, $100 \mu \mathrm{m}$. BMSCs, bone marrow mesenchymal stem cells; UCMSCs, umbilical cord mesenchymal stem cells.

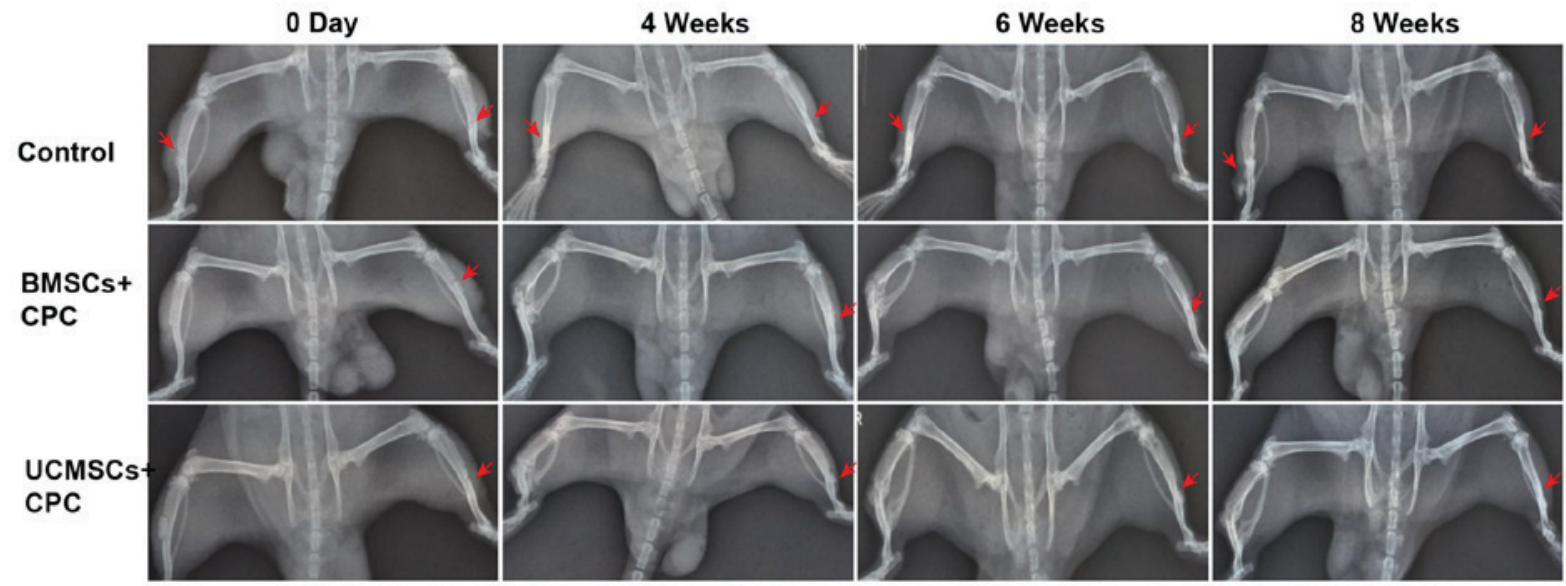

Figure 4. X-ray images of the rats at 0, 4, 6 and 8 weeks after implantation of CPC scaffold. For the control group, the right side of the rats was implanted with the cell-free CPC scaffold, whereas the left side was left untreated. The arrows indicate the area of the defect. CPC, calcium phosphate cement; BMSCs, bone marrow mesenchymal stem cells; UCMSCs, umbilical cord mesenchymal stem cells; 0 day, day of surgery; 4 weeks, 4 weeks after surgery.

increased expression of OPN and COL-1 was identified at the defect site of the experimental cell treatment groups compared with that in the CPC group (Fig. 5B and C). The H-scores for $\mathrm{OPN}$ in the BMSCs+CPC and the UCMSCs+CPC groups 


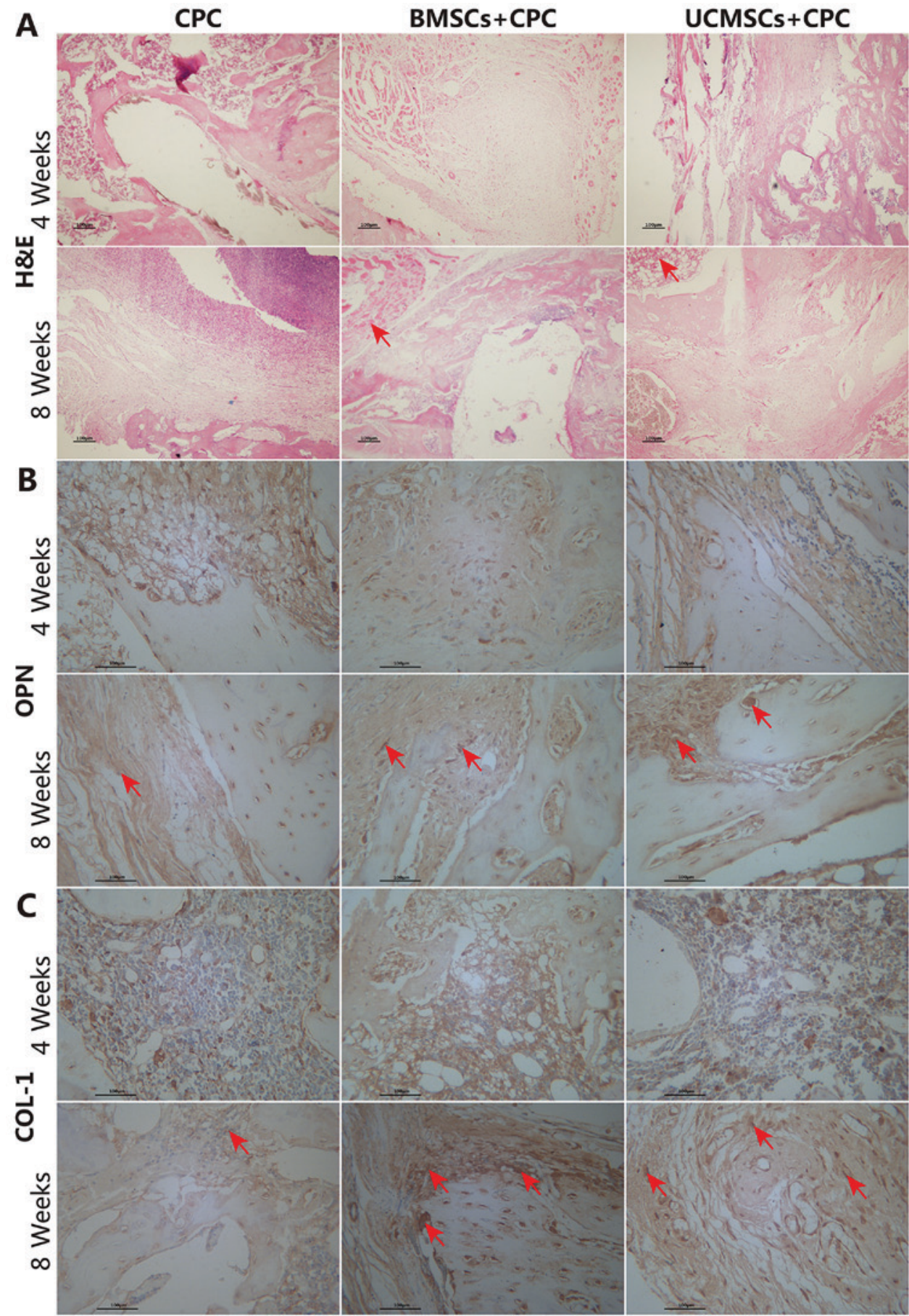

Figure 5. (A) H\&E staining and (B and C) IHC analysis of (B) OPN and (C) COL-1 were used to detect osteogenesis in the tibia defect rat model. The brown coloration represents positive staining for OPN and COL-1. The arrows indicate positive staining. Scale bars, $100 \mu \mathrm{m}$. IHC, immunohistochemical; OPN, osteopontin; COL-1, type I collagen; 4 weeks, 4 weeks after surgery; CPC, calcium phosphate cement.

were greater compared with that in the CPC group $(\mathrm{P}<0.05)$, while no significant differences were identified between the BMSCs+CPC and UCMSCs+CPC groups (Fig. 6A). Furthermore, the H-scores for COL-1 in the BMSCs+CPC and the UCMSCs+CPC groups were larger than that in the CPC group $(\mathrm{P}<0.05)$, while no significant difference was identified between the BMSCs+CPC and UCMSCs+CPC groups (Fig. 6B). These results indicated that BMSCs and UCMSCs did indeed accelerate the healing of the bone defect, although no significant differences were observed between the two cell types in terms of these effects.
Ectopic bone formation in the BMSCs + CPC and UCMSCs $+C P C$ groups in vivo. To further evaluate the osteogenic capacity of BMSCs and UCMSCs in vivo, engineering of cell-scaffolds or cell-free scaffolds were implanted under the skin of nude mice. The expression of Runx 2 and VEGF on the CPC was detected by immunofluorescence and immunohistochemical analyses. Runx 2 is the transcription factor that regulates the differentiation and maturation of osteogenic differentiation. For this reason, the expression of Runx 2 provides the hallmark of osteoblast differentiation; it is the earliest one that may be observed and the most specific marker gene in bone formation. 
A

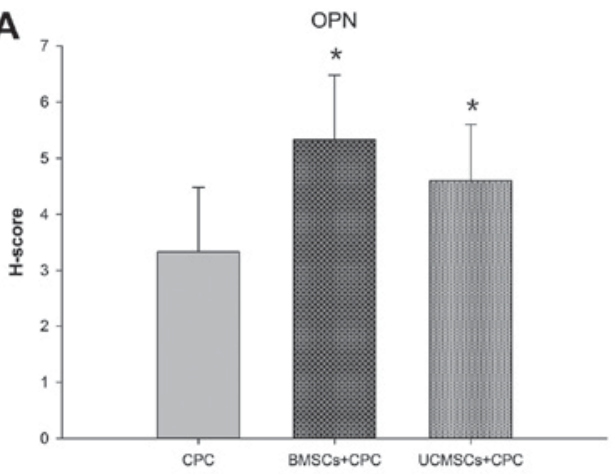

B

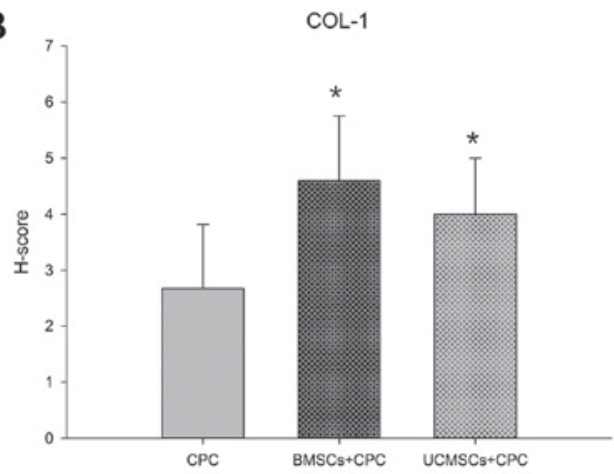

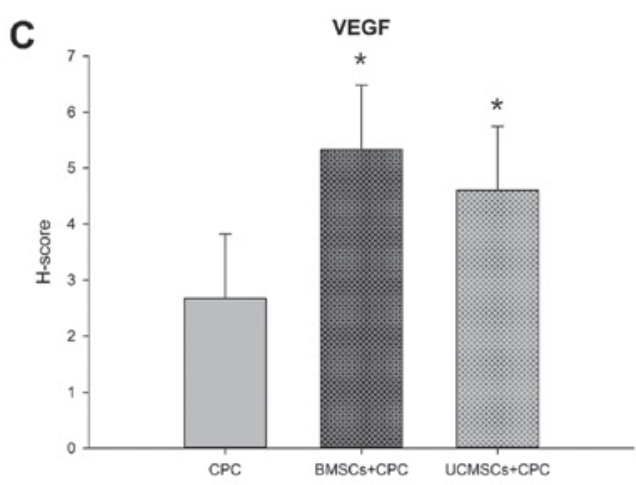

Figure 6. IHC staining scores for (A) OPN, (B) COL-1 and (C) VEGF at 8 weeks following surgery. ${ }^{*} \mathrm{P}<0.05$ vs. cell-free CPC group. IHC, immunohistochemical; OPN, osteopontin; COL-1, type I collagen; VEGF, vascular endothelial growth factor; CPC, calcium phosphate cement; BMSCs, bone marrow mesenchymal stem cells; UCMSCs, umbilical cord mesenchymal stem cells.

In the present study, a stronger intensity of fluorescence staining of Runx 2 was observed in the defect areas of the BMSCs+CPC and UCMSCs+CPC groups compared with that in the CPC group (Fig. 7A). An increase in the levels of VEGF was observed in all groups from 4-8 weeks post-surgery (Fig. 7B). At week 8 post-surgery, the $\mathrm{H}$-score of VEGF in the BMSCs+CPC and the UCMSCs+CPC groups were greater compared with that in the CPC group $(\mathrm{P}<0.05)$, while no significant differences were observed between the BMSCs+CPC group and the UCMSCs+CPC group (Fig. 6C). Therefore, these results suggested that the presence of BMSCs and UCMSCs led to an elevation in the rate of angiogenesis, and that this effect was not significantly different between the two cell types. The results of the ectopic bone formation experiments therefore exhibited the same trend as those of the experiments using the tibia defect model of bone regeneration.

\section{Discussion}

Bone tissue engineering involves the signaling molecule scaffold materials and the ideal seed cells $(12,27)$. Ideal seed cells should be safe and easy to obtain and to proliferate, and have the capability of undergoing osteogenesis. BMSCs and UCMSCs have been assessed for experimental periodontal tissue regeneration in a variety of animal models (28-31). A previous study by Shang et al (32) indicated that the osteogenic capability of UCMSCs was similar to that of BMSCs in an animal model of bone fracture healing. Furthermore, the above study demonstrated that UCMSCs express osteogenic differentiation markers, including ALP and Runx2. Given the limitations of BMSCs that were mentioned in the Introduction, the advantages of UCMSCs are even more pronounced. UCMSCs are derived from richly abundant sources, have lower viral infection rates and are not associated with any ethical obstacles. The aim of the present study was to compare the promotional effects of BMSCs and UCMSCs on bone regeneration, and to determine whether UCMSCs may be used as a novel cell source for bone regeneration. The results of the present study demonstrated that in vitro, BMSCs and UCMSCs have numerous properties in common, including adherence ability, proliferative capacity, immunophenotype and osteogenic differentiation ability.

MSCs promote bone repair when implanted locally, usually on a scaffold, e.g. CPC. Wang et al (12) have demonstrated that $\mathrm{CPC}$ has a good affinity for cell attachment without having any negative effects on cell viability. It was able to induce undifferentiated MSCs to become osteoblasts, thereby serving an osteoinductive function. Therefore, CPC was used as the carrier and scaffold of BMSCs and UCMSCs to repair bone defects in the present study.

Zhang et al (33) investigated the proliferative and osteogenic potential of MSCs from human fetal BMSCs, human UCMSCs, as well as human adult adipose tissue MSCs and BMSCs, in in vitro and in vivo models of ectopic bone formation. In the present study, in vivo experiments of ectopic bone formation and tibia bone defect were performed. Osteogenesis-associated protein markers, including OPN, COL-1, Runx2 and VEGF, were also detected. Therefore, the present study was more 


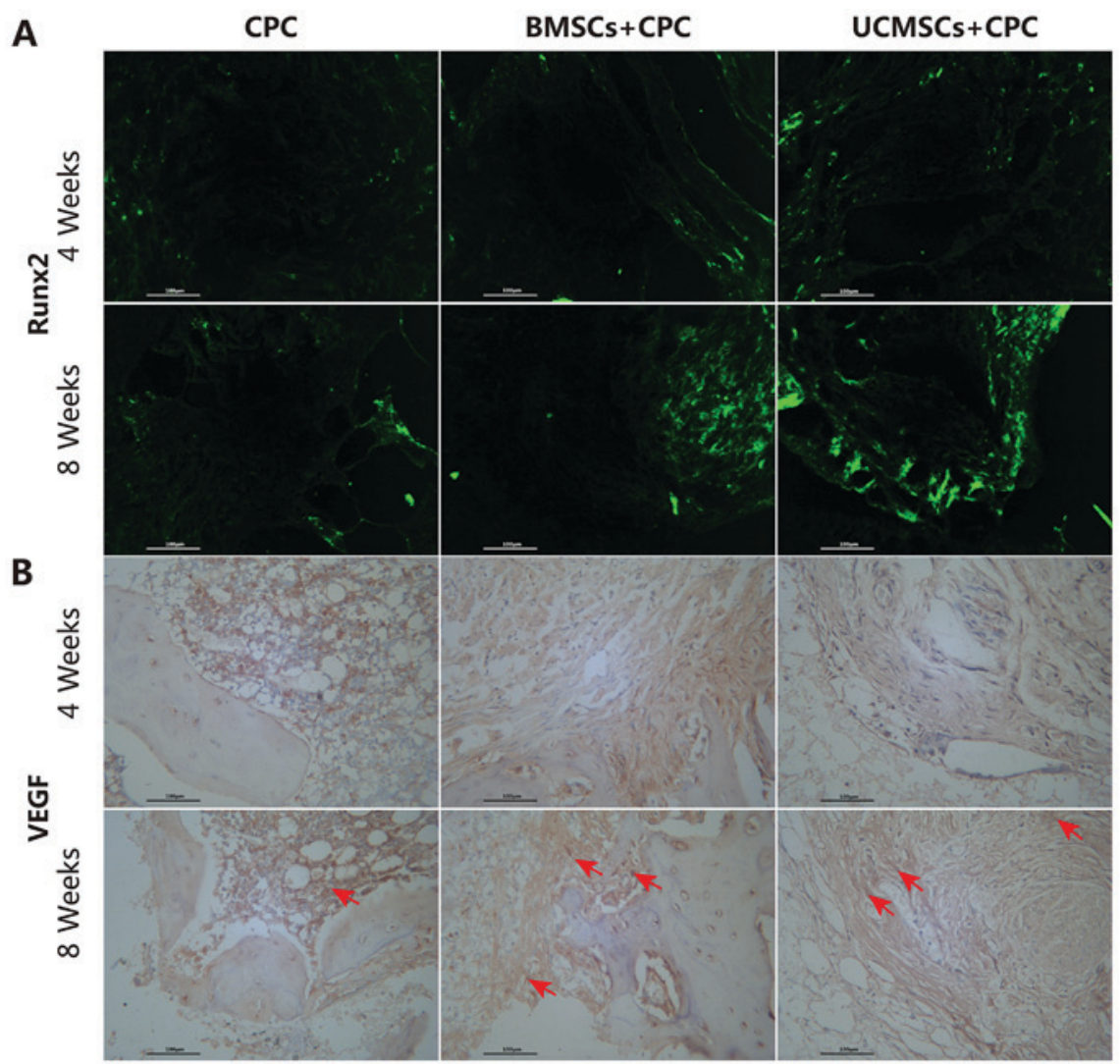

Figure 7. Ectopic bone formation of BMSCs and UCMSCs in nude mice. (A) Immunofluorescence assays of Runx 2 and (B) IHC staining of VEGF are shown. The green fluorescence represents Runx 2 and the brown coloration represents VEGF. The arrows indicate positive staining. Scale bars, $100 \mu \mathrm{m}$. IHC, immunohistochemical; Runx2, Runt-related transcription factor 2; VEGF, vascular endothelial growth factor; BMSCs, bone marrow mesenchymal stem cells; UCMSCs, umbilical cord mesenchymal stem cells; CPC, calcium phosphate cement.

comprehensive and clinical than the previous one. Regarding the choice of animal model, nude mice are ideal for assessing ectopic bone formation, as these immunocompromised animals lack a thymus and do not reject the implant, so that the cells may easily survive after transplantation $(34,35)$. However, rats are used for the classic animal model of bone defect $(36,37)$. In the present study, the X-ray results revealed that the application of BMSCs+CPC and UCMSCs+CPC in the tibia defect model led to improved levels of osteogenesis compared with that in the CPC only group. OPN and COL-1 serve as early markers of osteodifferentiation, whereas Runx2 is a key transcription factor required for bone formation $(23,38)$. Achieving increased expression levels of OPN, COL-1 and Runx2 is an essential prerequisite for bone formation. Angiogenesis is postulated to have an essential role in new bone formation, from the perspective of enhancing bone repair directly or indirectly by promoting angiogenesis and bone metabolism $(23,39,40)$. In the present study, the increase in the expression levels of OPN and COL-1 indicated that the BMSCs+CPC and UCMSCs+CPC treatments accelerated the bone regeneration in the rat model of tibia bone defect, and no significant differences were observed comparing between the two groups. These results were consistent with those of the X-ray analysis. In the ectopic bone formation experiments, the expression levels of Runx2 and VEGF were increased in all groups from 4-8 weeks. These results also suggested that the BMSCs and UCMSCs accelerated the regeneration of the bone defect, while no significant differences were observed between the two cell types. However, more indicators associated with angiogenesis are required to be detected in future studies, including CD31, which is a marker of microvessel density. The combination of VEGF and CD31 would more clearly indicate angiogenesis in the bone defect areas.

In conclusion, all of the results of the present study demonstrated that BMSCs and UCMSCs improve bone regeneration and angiogenesis in tibia defect and ectopic bone formation models, and that UCMSCs may be used as an alternative to replace BMSCs as an ideal type of seed cell for bone tissue engineering. The present study may provide a novel lead for the selection of seed cells for bone tissue engineering, and also enhances the current knowledge regarding the treatment of bone defects. However, apart from the MSC markers assessed in vitro, the expression of other types of $\mathrm{CD}$ was not assessed, and the detailed mechanisms of the osteogenesis of BMSCs and UCMSCs still require further investigation; those will present the next challenge in future studies by our group.

\section{Acknowledgements}

Not applicable.

\section{Funding}

This study was supported by the Natural Science Foundation of Tianjin, China (grant no. 14JCYBJC43900). 


\section{Availability of data and materials}

The datasets used and/or analyzed during the current study are available from the corresponding author on reasonable request.

\section{Authors' contributions}

JM and GZ conceived and designed the study. QW, GZ, ZJX and JMZ performed the experiments. QW wrote the paper. QW, JM and GZ reviewed and edited the manuscript. All authors have read and approved the manuscript.

\section{Ethics approval and consent to participate}

All animal experiments were reviewed and approved by the Animal Experimentation Ethics Committee of Institute of Radiation Medicine Chinese Academy of Medical Sciences (Tianjin, China).

\section{Patient consent for publication}

Not applicable.

\section{Competing interests}

The authors declare that they have no competing interests.

\section{References}

1. Szpalski C, Barr J, Wetterau M, Saadeh PB and Warren SM: Cranial bone defects: Current and future strategies. Neurosurg Focus 29: E8, 2010.

2. Terella A, Mariner P, Brown N, Anseth K and Streubel SO Repair of a calvarial defect with biofactor and stem cell-embedded polyethylene glycol scaffold. Arch Facial Plast Surg 12: 166-171, 2010.

3. Meinel L, Betz O, Fajardo R, Hofmann S, Nazarian A, Cory E, Hilbe M, McCool J, Langer R, Vunjak-Novakovic G, et al: Silk based biomaterials to heal critical sized femur defects. Bone 39 922-931, 2006

4. Megerdichian S: Adipose-derived perivascular stem cells heal critical size mouse calvarial defects. UCLA. ProQuest ID: Megerdichian_ucla_0031N_11204. Merritt ID: ark:/13030/ m5fr24h4. Dissertations and Theses-Gradworks 2013. https://escholarship.org/uc/item/4zz5k216.

5. Wang S, Qu X and Zhao RC: Mesenchymal stem cells hold promise for regenerative medicine. Front Med 5: 372-378, 2011

6. Xu L, Liu Y, Sun Y, Wang B, Xiong Y, Lin W, Wei Q, Wang H He W, Wang B and Li G: Tissue source determines the differentiation potentials of mesenchymal stem cells: A comparative study of human mesenchymal stem cells from bone marrow and adipose tissue. Stem Cell Res Ther 8: 275, 2017.

7. Hoogduijn MJ and Dor FJ: Mesenchymal stem cells: Are we ready for clinical application in transplantation and tissue regeneration? Front Immunol 4: 144, 2013.

8. Lee OK, Kuo TK, Chen WM, Lee KD, Hsieh SL and Chen TH: Isolation of multipotent mesenchymal stem cells from umbilical cord blood. Blood 103: 1669-1675, 2004

9. Steinert AF, Rackwitz L, Gilbert F, Nöth U and Tuan RS: Concise review: The clinical application of mesenchymal stem cells for musculoskeletal regeneration: Current status and perspectives. Stem Cells Transl Med 1: 237-247, 2012.

10. Caplan AI: Review: Mesenchymal stem cells: Cell-based reconstructive therapy in orthopedics. Tissue Eng 11: 1198-1211, 2005.

11. Meyer U, Meyer TH, Handschel J and Wiesmann HP (eds.) Fundamentals of Tissue Engineering and Regenerative Medicine. Springer, Berlin Heidelberg, 2009.
12. Wang P, Liu X, Zhao L, Weir MD, Sun J, Chen W, Man Y and $\mathrm{Xu} \mathrm{HH}$ : Bone tissue engineering via human induced pluripotent, umbilical cord and bone marrow mesenchymal stem cells in rat cranium. Acta Biomater 18: 236-248, 2015.

13. Manferdini C, Cavallo C, Grigolo B, Fiorini M, Nicoletti A, Gabusi E, Zini N, Pressato D, Facchini A and Lisignoli G: Specific inductive potential of a novel nanocomposite biomimetic biomaterial for osteochondral tissue regeneration. J Tissue Eng Regen Med 10: 374-391, 2016.

14. Di Maggio N, Piccinini E, Jaworski M, Trumpp A, Wendt DJ and Martin I: Toward modeling the bone marrow niche using scaffold-based 3D culture systems. Biomaterials 32: 321-329, 2011.

15. Grover LM, Wright AJ, Gbureck U, Bolarinwa A, Song J, Liu Y, Farrar DF, Howling G, Rose J and Barralet JE: The effect of amorphous pyrophosphate on calcium phosphate cement resorption and bone generation. Biomaterials 34: 6631-6637, 2013.

16. Zhang J, Liu W, Schnitzler V, Tancret F and Bouler JM: Calcium phosphate cements for bone substitution: Chemistry, handling and mechanical properties. Acta Biomater 10: 1035-1049, 2014.

17. Calabrese G, Giuffrida R, Lo Furno D, Parrinello NL, Forte S, Gulino R, Colarossi C, Schinocca LR, Giuffrida R, Cardile V and Memeo L: Potential effect of CD271 on human mesenchymal stromal cell proliferation and differentiation. Int J Mol Sci 16: 15609-15624, 2015.

18. Vicari L, Calabrese G, Forte S, Giuffrida R, Colarossi C, Parrinello NL and Memeo L: Potential role of activating transcription factor 5 during osteogenesis. Stem Cells Int 2016: 5282185, 2016.

19. Ai J, Ebrahimi S, Khoshzaban A, Jafarzadeh Kashi TS and Mehrabani D: Tissue engineering using human mineralized bone xenograft and bone marrow mesenchymal stem cells allograft in healing of tibial fracture of experimental rabbit model. Iran Red Crescent Med J 14: 96, 2012.

20. Chen Y, Xu J, Huang Z, Yu M, Zhang Y, Chen H, Ma Z, Liao $\mathrm{H}$ and $\mathrm{Hu} \mathrm{J}$ : An innovative approach for enhancing bone defect healing using PLGA scaffolds seeded with extracorporeal-shock-wave-treated bone marrow mesenchymal stem cells (BMSCs). Sci Rep 7: 44130, 2017.

21. Tsuchida H, Hashimoto J, Crawford E, Manske P and Lou J: Engineered allogeneic mesenchymal stem cells repair femoral segmental defect in rats. J Orthop Res 21: 44-53, 2003.

22. Tanaka M, Fukushima N, Yamasaki F and Ohshima K: Primary hepatic extranodal marginal zone lymphoma of mucosa-associated lymphoid tissue type is associated with chronic inflammatory process. Open J Hematol 2010: 1-5, 2010.

23. Huang Z, Xu J, Chen J, Chen H, Wang H, Huang Z, Chen Y, $\mathrm{Lu} \mathrm{X}, \mathrm{Lu} \mathrm{F}$ and $\mathrm{Hu}$ J: Photoacoustic stimulation promotes the osteogenic differentiation of bone mesenchymal stem cells to enhance the repair of bone defect. Sci Rep 7: 15842, 2017.

24. Cha YJ and Shim HS: PD-L1 expression and $\mathrm{CD}^{+}$ tumor-infiltrating lymphocytes are associated with ALK rearrangement and clinicopathological features in inflammatory myofibroblastic tumors. Oncotarget 8: 89465-89474, 2017.

25. Freitas MA, Gomes Rde O, Soares BL, Artigiani Neto R, Montero EF and Martins JL: Effects of maternal ischemic preconditioning in the colon of newborn rats submitted to hypoxia-reoxygenation insult. Acta Cir Bras 29: 438-444, 2014.

26. Soslow RA, Dannenberg AJ, Rush D, Woerner BM, Khan KN, Masferrer J and Koki AT: COX-2 is expressed in human pulmonary, colonic, and mammary tumors. Cancer 89: 2637-2645, 2000.

27. Ye P, Yu B, Deng J, She RF and Huang WL: Application of silk fibroin/chitosan/nano-hydroxyapatite composite scaffold in the repair of rabbit radial bone defect. Exp Ther Med 14: 5547-5553, 2017.

28. Yang Y, Rossi FM and Putnins EE: Periodontal regeneration using engineered bone marrow mesenchymal stromal cells. Biomaterials 31: 8574-8582, 2010.

29. Tobita M, Uysal AC, Ogawa R, Hyakusoku H and Mizuno H: Periodontal tissue regeneration with adipose-derived stem cells. Tissue Eng Part A 14: 945-953, 2008.

30. Guo W, Chen L, Gong K, Ding B, Duan Y and Jin Y: Heterogeneous dental follicle cells and the regeneration of complex periodontal tissues. Tissue Eng Part A 18: 459-470, 2012.

31. Yan XZ, Yang F, Jansen JA, de Vries RB and van den Beucken JJ: Cell-based approaches in periodontal regeneration: A systematic review and meta-analysis of periodontal defect models in animal experimental work. Tissue Eng Part B Rev 21: 411-426, 2015. 
32. Shang F, Liu S, Ming L, Tian R, Jin F, Ding Y, Zhang Y, Zhang H, Deng $\mathrm{Z}$ and Jin Y: Human umbilical cord MSCs as new cell sources for promoting periodontal regeneration in inflammatory periodontal defect. Theranostics 7: 4370-4382, 2017.

33. Zhang ZY, Teoh SH, Chong MS, Schantz JT, Fisk NM, Choolani MA and Chan J: Superior osteogenic capacity for bone tissue engineering of fetal compared with perinatal and adult mesenchymal stem cells. Stem Cells 27: 126-137, 2009.

34. Zhang X, Li Y, Chen YE, Chen J and Ma PX: Cell-free 3D scaffold with two-stage delivery of miRNA-26a to regenerate critical-sized bone defects. Nat Commun 7: 10376, 2016.

35. Sensebé L and Fleury-Cappellesso S: Biodistribution of mesenchymal stem/stromal cells in a preclinical setting. Stem Cells Int 2013: 678063, 2013.

36. Wang J, Gao Y, Cheng P, Li D, Jiang H, Ji C, Zhang S, Shen C, Li J, Song Y, et al: CD31hiEmcnhi vessels support new trabecular bone formation at the frontier growth area in the bone defect repair process. Sci Rep 7: 4990, 2017.
37. Grewal BS, Keller B, Weinhold P and Dahners LE: Evaluating effects of deferoxamine in a rat tibia critical bone defect model. J Orthop 11: 5-9, 2013

38. Wang T, Yang X, Qi X and Jiang C: Osteoinduction and proliferation of bone-marrow stromal cells in three-dimensional poly (E-caprolactone)/hydroxyapatite/collagen scaffolds. J Transl Med 13: 152, 2015.

39. Geris L, Gerisch A, Sloten JV, Weiner R and Oosterwyck HV: Angiogenesis in bone fracture healing: A bioregulatory model. J Theor Biol 251: 137-158, 2008.

40. Tian XB, Sun L, Yang SH, Fu RY, Wang L, Lu TS, Zhang YK and Fu DH: Ectopic osteogenesis of mouse bone marrow stromal cells transfected with BMP 2/VEGF(165) genes in vivo. Orthop Surg 1: 322-325, 2009. 